\title{
ANALYSIS OF TIN BY CHARGED PARTICLE BEAMS: NUCLEAR REACTION ANALYSIS, NUCLEAR REACTION BROADENING AND RUTHERFORD BACKSCATTERING*
}

\section{H. ROTBERG}

Michigan Ion Beam Laboratory, Nuclear Engineering Department, University of Michigan, Ann Arbor, MI 48109 (U.S.A)
A. J. PERRY AND C. C. STRANDBERG
GTE Valenite Corporation, 1711 Thunderbird, Troy, MI 48084 (U.S.A.)
G. WAS

Michigan Ion Beam Laboratory, Nuclear Engineering Department, University of Michigan, Ann Arbor, MI 48109 (U.S.A)

(Received February 29, 1988; accepted May 8, 1988)

Charged particle beams have been used to extract data both on the composition and also on the thickness of two series of $\operatorname{TiN}_{x}$ films (about 5 and $2 \mu \mathrm{m}$ thick respectively), where $x$ varied from 0.3 to 1.0 . The technique used were nuclear reaction analysis (NRA), resonance nuclear reaction broadening (NRB) and Rutherford backscattering, together with Auger electron spectroscopy (AES). The data from the NRB depth profiles scale remarkably well with the results from AES. The NRA results have the greatest experimental uncertainty and also indicate a systematic error which is not understood. Both NRA and NRB can return reliable film thickness data. It is also demonstrated that TiC should not be used as a reference standard when analyzing TiN films by AES.

\section{INTRODUCTION}

The advent of plasma-enhanced physical vapor deposition (PVD) methods for making TiN films has made the whole range of nitrogen-to-titanium ratios available as it is known from the equilibrium phase diagram ${ }^{1-3}$. In addition, samples of superstoichiometric composition can also be made ${ }^{-7}$. It is apparent that the question of determining the composition of such PVD films has become more acute than was so in the case of films made by chemical vapor deposition which can be made reproducibly at or near to the stoichiometric composition with comparative ease.

In a previous paper ${ }^{8}$, a round robin study was reported where a number of surface analysis methods was employed. It was found, inter alia, that Auger electron spectroscopy (AES) could return reliable data following the analysis of Dawson and Tzatzov ${ }^{9}$ provided that the spectra were massaged such that the fine structure was

* Paper presented at the 15th International Conference on Metallurgical Coatings, San Diego, CA, U.S.A., April 11-15, 1988. 
folded in to make the near overlap of the nitrogen $K_{23} L_{23}$ and titanium $L_{3} M_{23} M_{23}$ appear as a true overlap peak; similarly, the cross-transition titanium $\mathrm{L}_{3} \mathrm{M}_{23}-$ nitrogen $\mathrm{NL}_{23}$ and titanium $\mathrm{L}_{3} \mathbf{M}_{23} \mathrm{M}_{45}$ overlap.

It has since been found ${ }^{10}$ that the AES analysis needs to be treated with care when working with single crystals or with very strongly textured films; this also renders questionable their use as standards in AES analysis. Other methods of analyzing TiN are used in other laboratories: Rutherford backscattering $(\mathrm{RBS})^{5,11^{-13}}$, nuclear reaction analysis $(\mathrm{NRA})^{5}$ and resonance nuclear reaction broadening (NRB) ${ }^{\mathbf{1 4} 15}$ have been used to analyze TiN. The first of these is generally only used on thin films of up to about $1.5 \mu \mathrm{m}$ thick and preferably on substrates of low atomic number.

The present work reports a study of the above three methods and a comparison with AES measurements. The samples used were the previous round robin ${ }^{8}$ set, termed series $A$ here, of five samples (some $5 \mu \mathrm{m}$ thick over the composition range $\operatorname{TiN}_{x}$ between 0.54 and 1.01) and a thinner series B of six films ${ }^{15}$ (some $2 \mu \mathrm{m}$ thick with $x$ values between 0.30 and 0.80 ) which had already been analyzed by NRB. This later series was kindly made available by $\mathrm{Mr}$. J. Valli.

As series $A$ had already been analyzed ${ }^{8}$ by AES, it was studied by NRB at Helsinki University. Conversely, already studied by NRB, series B was analyzed by AES at GTE Valenite. Finally, both series were analyzed by NRA and RBS at the Michigan Ion Beam Laboratory.

\section{ANALYTICAL METHODS}

\subsection{Rutherford backscattering}

The small quantity of a collimated incident particle beam such as helium which is backscattered from a sample surface has an energy defined by the mass of the scattering atoms; a typical computer-produced spectrum for a thin film is shown in Fig. 1(a). This gives a quantitative non-destructive simultaneous multi-element analytical tool which requires no standard. As the film to be analyzed is made thicker, there is an energy loss both in the incident as well as in the backscattered beams, so that the measured backscattered signal is broadened. In TiN samples of thickness greater than the order of about $1.5 \mu \mathrm{m}$, the titanium and nitrogen signals overlap (even if a substrate of low atomic mass such as beryllium is used) as shown in Fig. 1(b) for one of the present samples.

An alternative approach has been used here for the relatively thick film samples studied. The height of the titanium signal is compared with that expected from a pure titanium metal sample which can be calculated theoretically. An example is shown in Fig. 1(b) where the calculated curve for a stoichiometric film is compared with the experimental results from a film known to be stoichiometric. In practice, the difference in signal between the calculated metal spectrum and the measured film is attributed to lighter elements and assumed here to be only nitrogen. Using this approach, the total amount of incident beam particles has to be measured, in contrast to the foregoing thin film case. The overall uncertainty in the N:Ti ratio is estimated to be about $\pm 10 \%$. 


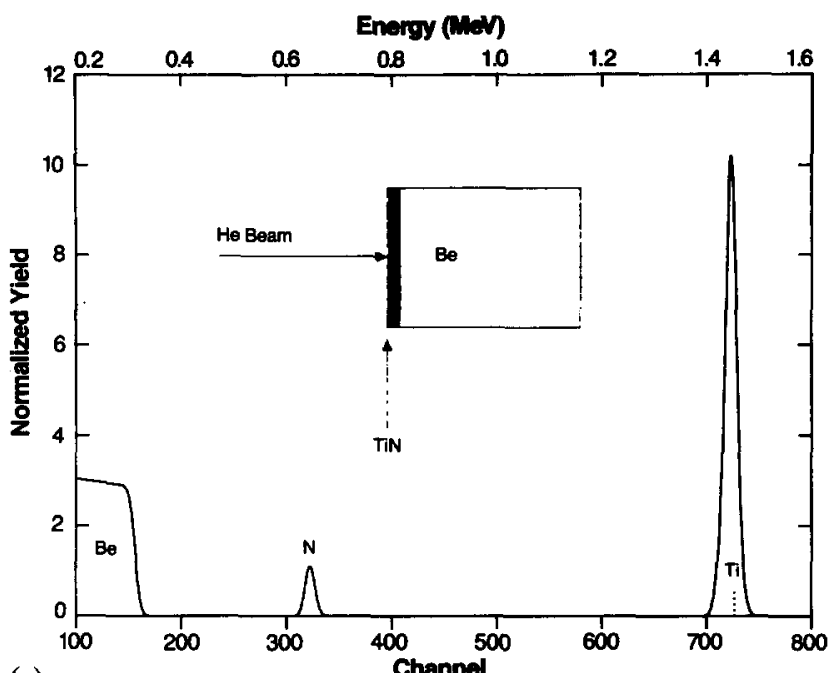

(a)

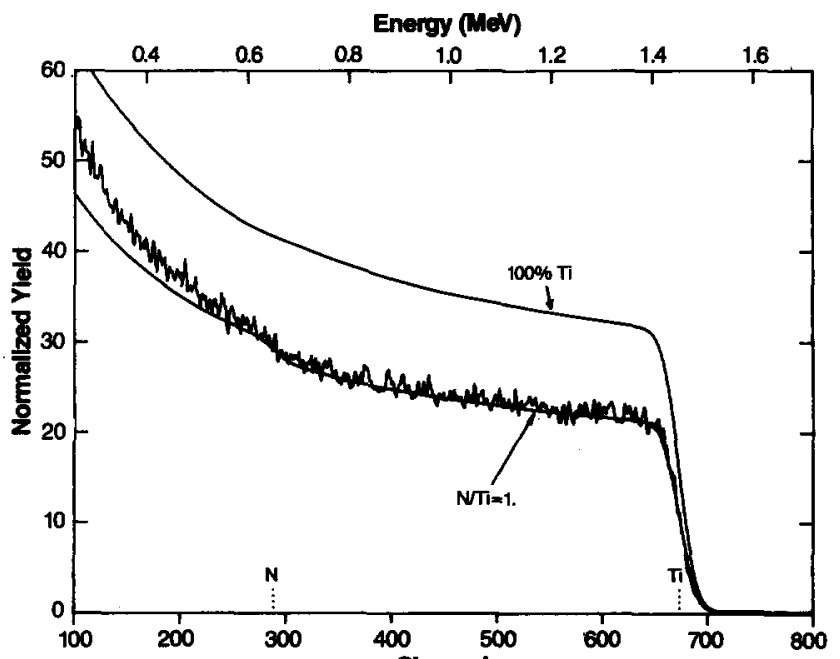

(b)

Fig. 1. RBS spectra for TiN films: (a) calculated for a thin film on a beryllium substrate and showing peaks due to the individual elements; (b) measured on a $5.3 \mu \mathrm{m}$ thick stoichiometric film (sample A5) together with the curves calculated for pure titanium and stoichiometric $\operatorname{TiN}_{1.0}$.

\subsection{Nuclear reaction analysis}

Another way of studying nitride films is through the use of direct nuclear reactions ${ }^{16-22}$. In this type of reaction, an incident beam of protons or deuterons interacts with the target nuclei producing highly unstable products which decay instantly into more stable structures by emitting characteristic radiations. A specific case is the deuteron reaction ${ }^{14} \mathrm{~N}(\mathrm{~d}, \alpha){ }^{12} \mathrm{C}$ where ${ }^{12} \mathrm{C}$ is formed from the abundant ${ }^{14} \mathrm{~N}$ isotope. This can be used to study the nitrogen in TiN. The $\alpha$ particles formed by the reaction are emitted at the two energy levels of about $10 \mathrm{MeV}$ and $7 \mathrm{MeV}$, and a typical spectrum is shown in Fig. 2. The reaction cross-section function is energy 
dependent and, since the beam loses energy inside the film, it has to be convoluted with the stopping cross-section in order to evaluate the yield of the reaction at every point, and the result integrated over the whole film. Since the stopping cross-section depends on the stoichiometry, which is to be determined from the experiment, the convolution is impossible. The situation is somewhat alleviated because the overall stopping power inside the film is not very strongly dependent on the stoichiometry and a rough knowledge of it would suffice for the calculations to be performed. However, to avoid this complication altogether, advantage is taken of the fact that the cross-section function exhibits a remarkably flat plateau ${ }^{17}$ from 2.0 to $2.4 \mathrm{MeV}$ at $135^{\circ}$. This energy range has been used to study $\mathrm{ZrN}$ films ${ }^{23}$. Since the reaction cross-section does not change with energy, the stopping cross-section is not needed and the stoichiometry does not need to be known a priori.

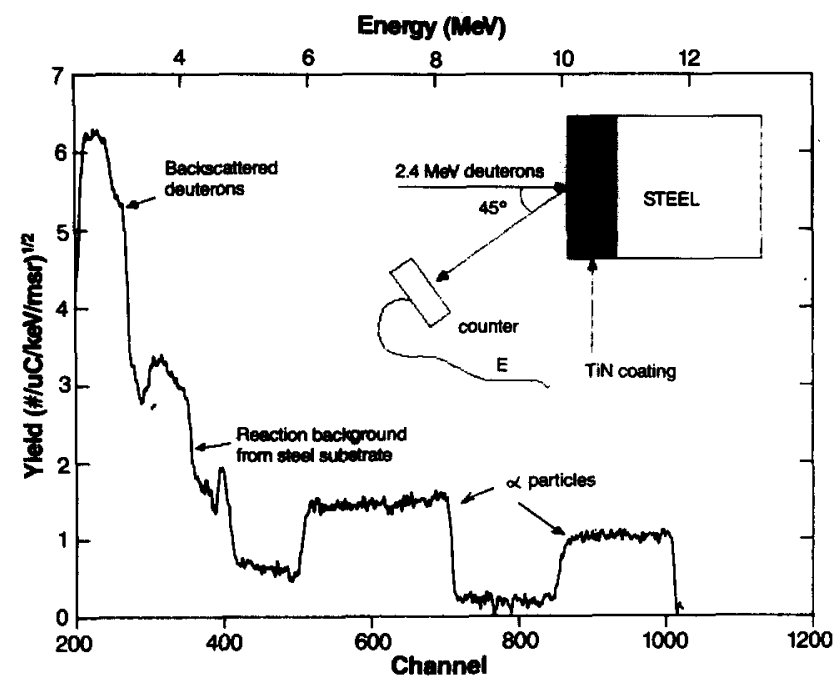

Fig. 2. NRA spectrum from a stoichiometric TiN film (sample A5) showing the two emitted $\alpha$ particle energy levels. The ordinate is given on a square root scale. A schematic diagram of the experimental arrangement is also shown.

The values for the cross-sections for both states $\alpha_{0}$ and $\alpha_{1}$ are given in ref. 17. The $\alpha_{1}$ state cross-section seems to be much more constant than the higher energy $\alpha_{0}$ which fluctuates around a mean value. Since the energy of the beam, in these thick samples, spans an interval much larger than the fluctuations, the cross-section can be assumed to be constant. Both states give the same amount of nitrogen in a very large number of samples measured. The $400 \mathrm{keV}$ energy range in which the crosssection is constant allows analysis of films of thicknesses up to about $5 \mu \mathrm{m}$ using this method.

The area under the $\alpha$ particle peaks is proportional to the number of nitrogen atoms per unit area of sample. If the sample thickness is measured (e.g. by the ball crater method) the number of atoms per unit volume can then be calculated and compared with that of a qualified standard so that the stoichiometry is obtained.

A useful feature is that the width of the $\alpha$ particle peaks is related to the film 
thickness and can be obtained from the spectrum if the film density is known, or assumed to be normal, and a rough knowledge of the stoichiometry is available.

The peak width is directly related to the density so it must be known as accurately as possible. The stoichiometry, however, will affect the peak width to a much lesser degree because, as mentioned above, the overall stopping power is not so strongly dependent on it. One can utilize values for stoichiometry given by one of the other techniques and obtain film thicknesses very accurately.

The accuracy in the $\mathrm{N}$ :Ti ratio is estimated to be $\pm 5 \%$. In the present work, sample A5 which is known ${ }^{8}$ to be stoichiometric was used as standard. The results obtained were the same irrespective of which $\alpha$ particle peak was used.

\subsection{Resonance nuclear reaction broadening}

This technique ${ }^{22}$ uses a nuclear reaction which resonates only at, and very close to, a specific energy. A particular reaction ${ }^{24}$ of the rare ${ }^{15} \mathrm{~N}$ isotope (natural abundance, $0.37 \%{ }^{25}$ ) resonates at $430 \mathrm{keV}$ under proton bombardment and can be applied to nitrogen in titanium. The reaction ${ }^{15} \mathrm{~N}(\mathrm{p}, \alpha \gamma){ }^{12} \mathrm{C}$ transforms the ${ }^{15} \mathrm{~N}$ into ${ }^{16} \mathrm{O}$ which decays into ${ }^{12} \mathrm{C}$ by emitting an $\alpha$ particle and a $\gamma$ ray. Within a film, the energy loss by the proton in transit can be compensated for by raising the incident particle energy. The reaction then occurs at the depth in the film specified by that energy. The $\gamma$ rays are collected and then deconvoluted to give a profile from which the composition can be quantified by reference to a standard. A convenient aspect is that the film thickness need not be known, and indeed it can be measured if less than the penetrating power of the proton source. The resulting nitrogen distribution plots are typified by those shown in Fig. 3 and taken from the present series B samples (redrawn from curves kindly made available by Dr. J. M. Molarius).

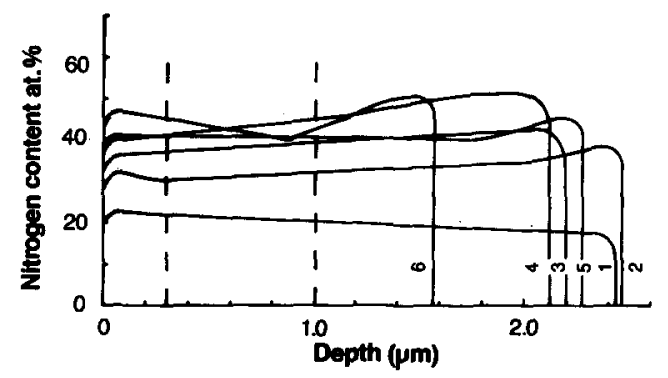

Fig. 3. NRB profiles of the present series B samples, redrawn from curves supplied by Dr. J. M. Molarius.

In the present work, the series A samples were analyzed at Helsinki University by Dr. A. Antilla. The present writers are greatly indebted to him for his support.

\subsection{Auger electron spectroscopy}

The methods followed here were identical with those used in our previous study $^{8}$. The one sample (A5) then shown to be stoichiometric and of nearly random crystal orientation from the round robin series $A$ was used as standard for series $B$, which had already been analyzed ${ }^{15}$ by NRB.

As is apparent from Section 1, the question of a qualified standard for AES 
measurements is non-trivial. Ideally a chemically qualified randomly oriented polycrystalline material should be used. As a subsidiary study here, a data analysis was carried through using $\mathrm{TiC}$ as a reference standard as this has been done by others ${ }^{13,26}$. The present results which are included in Fig. 4(b) indicate clearly that such usage leads to incorrect results (as does the use of a TiN single crystal ${ }^{10}$ ). The reason is that the titanium peak shape changes with chemical environment, as is well established $^{27}$.

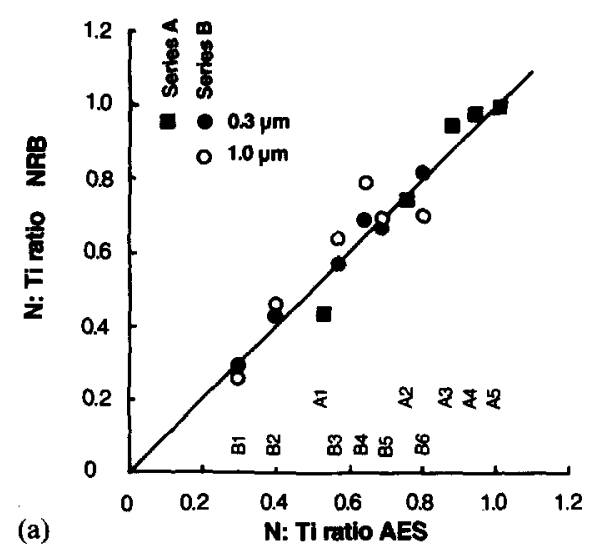

Fig. 4. (a) A comparison of AES data with NRB data for series A and series $B$. In the latter case the composition is not constant with depth (see Fig. 3) so a comparison is made at depths of $0.3 \mu \mathrm{m}$ and $1.0 \mu \mathrm{m}$ respectively; (b) A comparison data measured from series A by AES and EPMA ${ }^{8}$ and from series $B$ by AES and NRB at a depth of $0.3 \mu \mathrm{m}$. Additional data are shown $(O, \square)$ indicating the compositions derived from the present AES spectra had a TiC standard been used for comparison.

\section{RESULTS}

\subsection{Auger electron spectroscopy and nuclear reaction broadening}

In series B, all the nitrogen profiles from the NRB results (Fig. 3) show a slight fall near the surface; it is possible that this is due to surface oxidation of the type discussed by Ernsberger et al. ${ }^{28}$ In addition and more significantly, the samples were not always perfectly homogenous in nitrogen content through their thickness profiles at greater film depths. In the original study by Valli et al. ${ }^{15}$ on these samples, the composition values quoted had been taken at a depth of $1 \mu \mathrm{m}$. To make a data comparison valid here, the nitrogen content indicated by the NRB curves was selected at a depth of $0.3 \mu \mathrm{m}$ as corresponding approximately to the penetration depth of AES (after sputter cleaning). At this depth the NRB profiles are also reasonably flat (Fig. 3). The AES and NRB results are compared in Fig. 4(a). Whereas the comparison made with the original NRB composition values taken at $1.0 \mu \mathrm{m}$ (open symbols) is not very satisfactory, a comparison at the depth of $0.3 \mu \mathrm{m}$ (full symbols) allows a quite satisfactory agreement. This underlines the need to consider the depth of material sampled when comparing results taken with different techniques if composition variations are suspected.

Agreement between AES and NRB for sample series A is also satisfactory except for sample Al at which is not understood. Such generally good agreement 
had also been found when comparing the results ${ }^{8}$ from series $A$ with electron probe microanalysis (EPMA) results. For completeness, Fig. 4(b) includes the round robin comparison of series A by AES and EPMA and the present series B compared by AES with NRB at a $0.3 \mu \mathrm{m}$ sampling depth.

\subsection{Film thickness}

As indicated above, the film thickness can be determined in NRA measurements from the width of the $\alpha$ peaks. In the NRB method, the film thickness is given by the cut-off point in the nitrogen profiles. Both of the methods can be applied up to the penetration depths of the incident beams and exit depths of the particles produced; NRA can thus be used over a greater range of film thicknesses. As a third method, the film thickness can also be determined by the ball polishing method which is frequently used in hard coating studies.

The interpretation of the $\alpha$ peak width to determine film thickness from NRA requires the film density to be known. These data are available for series $B$, as measured by X-ray diffraction for an earlier study of Young's modulus ${ }^{29}$; the results of the thickness analysis are given in Fig. 5. This film series is too thick to be penetrated through to the substrate by the proton beam used, so the thickness could not be measured by NRB. In contrast, series A thicknesses could be measured with the latter technique, whereas those of series B could not because the film density was not known.

The thickness results returned for series A by NRA and for series B by NRB are given in Fig. 5 as a function of the values derived from the ball polishing method. The agreement is satisfactory.

\subsection{Nuclear reaction analysis and Rutherford backscattering}

The results of the extension of the study to include these two techniques are shown in Fig. 6 for both sample series. For convenience, the results of both methods
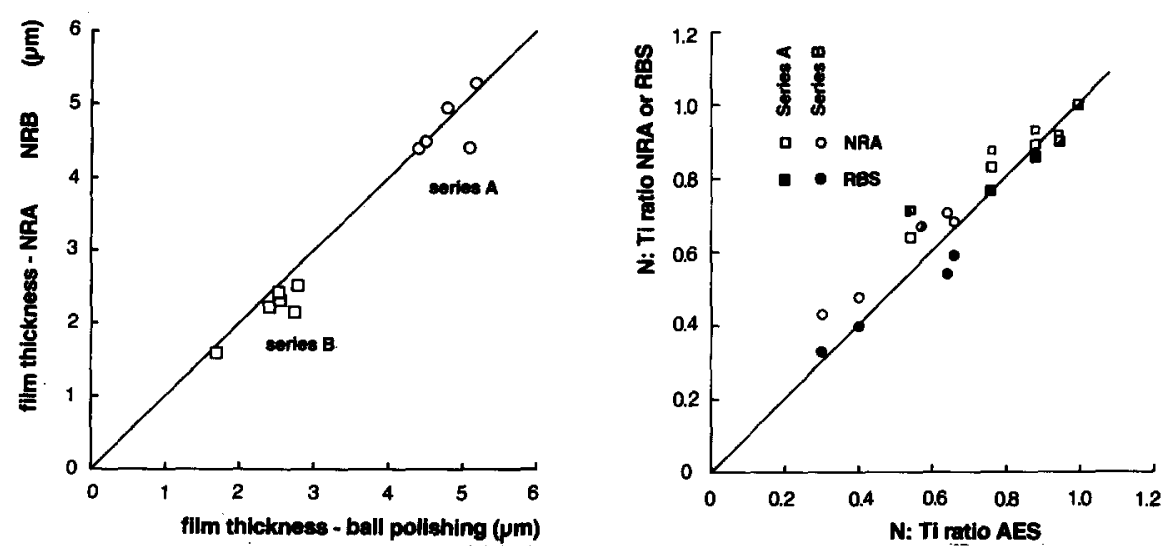

F1g. 5. Film depth obtained trom both sample series A and B as determined by NRA and NRB respectively and by the ball polishing method.

Fig. 6. A comparison between data measured by RBS and NRA with AES for series A and series B respectively. The NRA data for series $A$ are shown uncorrected (smaller squares) and corrected (larger squares) for density effects on the film thickness determination. 
are compared with the AES results because all our work to date has indicated the general reliability of this analytical method as it is applied here; this is the significance of the results presented in Fig. 4(b).

For series A, the agreement between NRA and RBS on the one hand and AES on the other appears perfectly acceptable (except for film A1) although the NRA data appear high.

As the density of the series B films was not known, it was taken to be equal to that of stoichiometric TiN which leads to a systematic error in the NRA data for this series, all of which indeed appear to indicate high N:Ti ratios. To assess the effect of this density approximation on the data for series $\mathrm{B}$, the composition results for series A were recalculated using the density of stoichiometric $\mathrm{TiN}$ in deriving the film thickness. The results are included in Fig. 6 (small open squares) and do show an increase in the apparent N:Ti ratio, confirming that the ratios derived here for series B could be high. However, taking all the NRA data for both series together irrespective of the density question, there does appear to be a systematic deviation to higher N:Ti ratios.

Referring to the nitrogen profiles in Fig. 3 as determined by NRB, it might be expected that NRA would indicate generally the same or a greater N:Ti ratio than RBS as more of the film is sampled by the former. This agrees with the general trend in Fig. 6, but not with the detailed profiles. Further, the same argument would lead to both techniques' again indicating the same or a greater $\mathrm{N}$ :Ti ratio than AES with its low sampling depth, again because many of the samples show the nitrogen level to increase with depth from the surface. This is not the trend which is observed.

\section{CONCLUSIONS}

The compositions of two series of $\mathrm{TiN}_{x}$ films, encompassing a wide range of stoichiometry $x$, has been studied by four analytical methods. These show that data taken from NRB depth composition profiles scale remarkably well with results from AES, provided that the low penetration depth of the latter is taken into account.

Reasonable agreement between the RBS data and the data found by AES (and NRB and EPMA) is noted but there appears to be a somewhat greater experimental uncertainty. RBS has a great advantage over these other methods because it requires no standard and the film thickness does not need to be known.

NRA was considered to have the greatest experimental uncertainty and this is borne out by the present results. In addition, the results indicate a systematic error which is not understood at the present time.

Measurements of film thickness by NRB (applicable to the thinner series B films only) and NRA (for series A where the density was known) are in good agreement with results from the ball polishing method.

As a subsidiary result, it is demonstrated that $\mathrm{TiC}$ should not be used as a reference standard when analyzing TiN films by AES.

REFERENCES

1 L. A. McClaine and C. P. Coppel, AFNL-TR-65-299, 1965. 
2 E. Etchessar, J. P. Bars and J. Debuigne, J. Less-Common Met., 134 (1987) 123.

3 L. E. Toth, Transition Metal Carbides and Nitrides, Academic Press, New York, 1971, p. 88.

4 S. Schiller, G. Beister and W. Sieber, Thin Solid Films, 11 (1984) 259.

5 J. P. Noel, D. C. Houghton, G. Este and F. R. Shephard, J. Vac Sci. Technol. A, 2 (1984) 284.

6 I. N. Martev, E. I. Grigorov, I. G. Petrov and E. Dynowski, Thin Solid Films, 131 (1985) 303.

7 E. O. Ristolainen, J. M. Molarius, A. S. Korhonen and J. K. Lindroos, J. Vac. Sci. Technol. A, 5 (1987) 2184.

8 A. J. Perry, C. Strandberg, W. D. Sproul, S. Hofmann, C. Ernsberger, J. Nickerson and L. Chollet, Thin Solid Films, 153 (1987) 169.

9 P. T. Dawson and K. K. Tzatzov, Surf. Sci., 149 (1985) 105.

10 C. Strandberg and A. J. Perry, Surf. Interface Anal., 12 (1988) 131.

11 B. J. Burrow, A. E. Morgan and R. C. Ellwanger, J. Vac. Sci. Technol. A, 4 (1986) 2463.

12 K. Y. Ahn, W. Wittmer and C. Y. Ting, Thin Solid Films, 93 (1982) 397.

13 N. Kaufherr, G. R. Fenske, D. E. Busch, P. Lin, C. Deshpandey and R. F. Bunshah, Thin Solid Films, 153 (1987) 149.

14 J. M. Molarius, A. S. Korhonen and E. O. Ristolainen, J. Vac. Sci. Technol A, 3(1985) 2419.

15 J. Valli, J. M. Molarius and A. S. Korhonen, Thin Solid Films, 154 (1987) 351.

16 G. Amsel, Nucl. Instrum. Methods, 92 (1971) 481.

17 G. V. Debras and G. Deconnincix, J. Radioanal. Chem., 38 (1977) 196.

18 W. K. Chu, J. W. Mayer, M. A. Nicolet, T. M. Buck, G. Amsel and F. Eisen, Thin Solid Films, 17 (1973) 1 .

19 T. Lenz, H. Baumann and F. Rauch, Nucl. Instrum. Methods B, 28 (1987) 280.

20 C. R. Gosset, Nucl. Instrum. Methods, 168 (1980) 217.

21 A. Barcz, A. Turos and L. Wielunski, J. Radioanal. Chem., 31 (1976) 227.

22 J. Hirvonen and A. Antilla, Appl. Phys. Lett., 35 (1979) 703.

23 J. C. B. Simpson, L. G. Earwaker and M. N. Khan, Nucl. Instrum. Methods B, 24-25 (1986) 701.

24 A. Antilla, J. Räisänen and J. Keinonen, Appl. Phys. Lett., 42 (1983) 498.

25 CRC Handbook of Chemistry and Physics, CRC Press, Boca Raton, 66th edn., 1985, p. B235.

26 T. Sekine, Y. Ando and H. Tokumasu, J. Vac. Sci. Technol. A, 4(1986) 1557.

27 B. J. Burrow, A. E. Morgan and R. C. Ellwanger, J. Vac. Sci. Technol A, 4 (1986) 2463.

28 C. Ernsberger, J. Nickerson, A. E. Miller and J. Moulder, J. Vac. Sci. Technol. A, 3 (1985) 2415.

29 E. Török, A. J. Perry, L. Chollet and W. D. Sproul, Thin Solid Films, 153 (1987). 
